\title{
An Eyetracker Study of the Haptic Cuing of Visual Attention
}

\author{
Chanon M. Jones, ${ }^{*}$ J. Jay Young, ${ }^{*}$ Rob Gray, ${ }^{\dagger}$ Charles Spence, ${ }^{\dagger}$ and Hong Z. Tan ${ }^{*}$ \\ (*) Haptic Interface Research Laboratory, Purdue University, West Lafayette, IN, USA \\ (†) Perception \& Action Laboratory, Arizona State University East, Mesa, AZ, USA \\ (t) Crossmodal Research Laboratory, Oxford University, Oxford, UK \\ E-mail: \{cmjones, hongtan\}@purdue.edu,robgray@asu.edu, charles.spence@psy.ox.ac.uk
}

\begin{abstract}
We investigated the haptic cuing of visual attention using spatially-predictive (75\% valid) and spatiallynonpredictive (25\%) haptic cues. The participants performed a visual change detection task immediately following a haptic spatial cue whose location corresponded to one of the four visual quadrants. The participants were explicitly instructed to use the spatially-predictive haptic cues but to try and ignore the spatially-nonpredictive cues. In addition to reaction time (RT) data, we recorded participants' eyeposition in order to provide a direct measure of overt visual attention. The results indicated that the spatially-predictive haptic cues reduced the amount of time taken to detect the visual changes, as expected. The spatially-nonpredictive cues increased visual search latencies, indicating that the cues could not be ignored completely. There was also evidence that haptic cuing served to alert the participants resulting in an overall reduction of response latencies.
\end{abstract}

\section{Introduction}

Many recent studies have demonstrated strong crossmodal links in spatial attention between vision and touch [1-5]. For example, visual spatial attention can be primed and re-directed as a result of receiving a vibrotactile cue. Applications utilizing this form of multisensory integration provide performance enhancement as the result of an intuitive mapping between proximal vibrotactile cues and distal visual events. What remains to be determined, however, is how strong this form of attentional link really is. The current study sought to investigate the extent to which the priming of visual spatial attention by vibrotactile cues is voluntary or automatic. This was accomplished by utilizing an eyetracker (ISCAN Inc., ETL-400) to monitor the eye position of participants, and by recording manual RT data (as obtained in our previous studies).

\section{Methods}

Ten participants (4 males, 6 females; 21-26 years old, averaging 23 years old) took part in the study as paid volunteers. All had normal or corrected vision and reported no known abnormalities with tactile perception on their backs.

Haptic cuing was provided via four tactors on the back of an office chair (see Fig.1a). A $60 \mathrm{~ms} 290 \mathrm{~Hz}$ sinusoidal pulse was sent to one of the tactors at an intensity that could be clearly felt on the participant's back through any clothing that they happened to be wearing. The visual stimuli, based on the flicker paradigm developed in [6] for studies of change blindness, consisted of 12 white rectangular elements, 3 per quadrant, on a black background (Fig.1b). Two alternating scenes, each lasting for $80 \mathrm{~ms}$ and differing only in the orientation of one of the rectangular elements, were presented. A $200 \mathrm{~ms}$ blank screen was inserted between alternating scenes to eliminate any visual motion transients (as they are known to facilitate performance on this kind of task). The sequence was repeated until the participant detected the changing element. Further details on experimental setup can be found in [3].

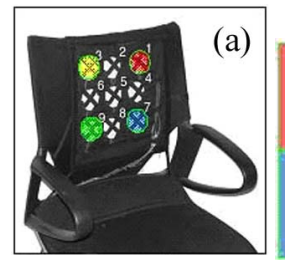

(b)

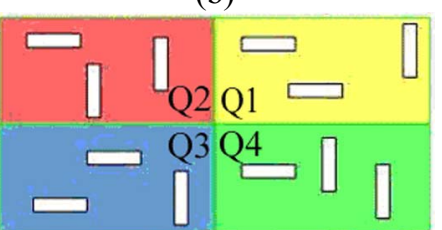

Figure 1. Schematic illustration of the layout of (a) tactor array, and (b) visual stimuli and the four quadrants. The color code indicates the correspondence between the visual quadrants and the tactors. During the experiments, the background of the computer screen was black. 


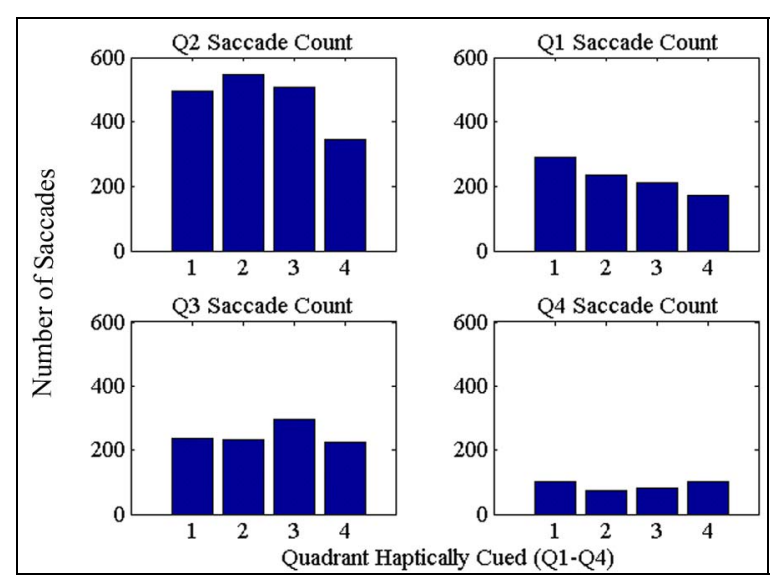

Figure 2. Pooled initial saccadic responses in the spatially-nonpredictive cuing condition.

The participants were presented with two experimental conditions (order counterbalanced). In the $75 \%$-validity condition, the quadrant in which the haptic cue was presented coincided with that of the visual changing element on $75 \%$ of the trials. Consequently, the haptic cues were spatially predictive and the participants were encouraged to utilize the haptic cues in order to aid their performance on the visual change detection task. In the 25\%-validity condition, the correspondence between the quadrants of the haptic cue and the visual change was at chance level and the participants were encouraged to ignore the cues. Baseline measures were also taken with no haptic cuing. Each participant completed 1320 trials.

For each participant, RT and eye-position data formed 16 conditions corresponding to the 16 cuechange quadrant pairs. RTs for trials with valid (cued quadrant $=$ visual change quadrant) and invalid cues were analyzed separately. Trials on which participants failed to locate the visual change correctly $(<7 \%)$ were discarded. Cuing effect was determined by subtracting the baseline RTs from those obtained with haptic cues (Delta RT).

\section{Results and Conclusion}

The mean RT in the baseline condition was $1520 \mathrm{~ms}$ (Q1), 1300ms (Q2), 1601ms (Q3), and 1678ms (Q4). It therefore appeared that participants tended to search the visual display serially starting at the top left quadrant in the order of Q2, Q1, Q3 and Q4 in the tactor-off condition. The participants responded an average of $467 \mathrm{~ms}$ faster following valid cuing in the $75 \%$-validity condition, as was expected; and $82 \mathrm{~ms}$ faster following invalid cues. Although we would expect a slight increase in mean RT with invalid cues, it is quite possible that the very presentation of the haptic cues served to alert the participants and the resulting speed-up in RT masked the slight increase in RT due to invalid cues [7].

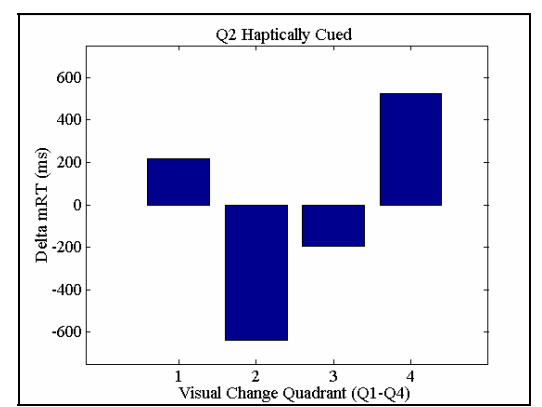

Figure 3. Change in mean RT for one participant in the $25 \%$-validity condition. Shown are the changes in RT (as compared to baseline) for visual changes in the four quadrants when Q2 was cued haptically.

The more interesting data came from the spatiallynonpredictive cuing condition. The mean RT increased following both valid and invalid cues by 218 and $41 \mathrm{~ms}$, respectively, indicating that the participants were unable to ignore the haptic cues completely, despite explicit instructions for them to do so. From the number of initial saccades made following haptic cuing (Fig. 2), it appears that the number of saccades per quadrant was highest when the quadrant was cued haptically. Moreover, some participants detected changes faster if the visual change occurred in a quadrant that was adjacent to that cued haptically than if the change quadrant was diagonally-opposite to the one cued (see Fig. 3). For example, when Q2 was cued, there was a decrease in RT for visual changes in Q3, a moderate increase in RT for changes in Q1, and the largest increase in RT occurred for changes in Q4.

The results obtained in the present study, especially the RT and eye-position data from the spatiallynonpredictive cuing condition, demonstrates that the crossmodal spatial attentional link between touch and vision is a strong one and cannot easily be ignored even when it is known that the haptic cues were not spatially informative, and when the relationship between the position of the haptic cues within the display and the visual scene is purely symbolic.

\section{References}

[1] R. Gray and H. Z. Tan, "Dynamic and predictive links between touch and vision," Experimental Brain Research, vol. 145, pp. 50-55, 2002

[2] H. Z. Tan, R. Gray, J. J. Young, and R. Traylor, "A haptic back display for attentional and directional cueing," Haptics-e: The Electronic Journal of Haptics Research, vol. 3, pp. 20 pp, 2003.

[3] J. J. Young, H. Z. Tan, and R. Gray, "Validity of haptic cues and its effect on priming visual spatial attention," Proceedings of the 11th International Symposium on Haptic Interfaces for Virtual Environment and Teleoperator System, pp. 166-170, 2003.

[4] C. Spence and J. Driver, "Crossmodal Space and Crossmodal Attention." Oxford University Press, 2004.

[5] A. Gallace, M. Auvray, H. Z. Tan, and C. Spence, "When visual transients impair tactile change detection: A novel case of crossmodal change blindness?," Neuroscience Letters, vol. 398, pp. 280-285, 2006.

[6] R. A. Rensink, "Visual search for change: A probe into the nature of attentional processing," Visual Cognition, vol. 7, pp. 345-376, 2000.

7] A. Gallace, H. Z. Tan, and C. Spence, "The body surface as a communication system: The state of the art after 50 years," to appear in Presence: Teleoperators and Virtual Environments. 